\title{
Roles of Nuclear Pores and Nucleo-cytoplasmic Trafficking in Plant Stress Responses
}

\begin{abstract}
Yu Yang ${ }^{1}$, Wei Wang ${ }^{2,3}$, Zhaoqing Chu ${ }^{2,3}$, Jian-Kang Zhu ${ }^{1,4}$ and Huiming Zhang ${ }^{1 *}$
${ }^{1}$ Shanghai Center for Plant Stress Biology, Shanghai Institutes for Biological Sciences, Chinese Academy of Sciences, Shanghai, China, ${ }^{2}$ Shanghai Key Laboratory of Plant Functional Genomics and Resources, Shanghai Chenshan Botanical Garden, Shanghai, China, ${ }^{3}$ Shanghai Chenshan Plant Science Research Center, Chinese Academy of Sciences, Shanghai, China, ${ }^{4}$ Department of Horticulture and Landscape Architecture, Purdue University, West Lafayette, IN, USA
\end{abstract}

The nuclear pore complex (NPC) is a large protein complex that controls the exchange of components between the nucleus and the cytoplasm. In plants, the NPC family components play critical roles not only in essential growth and developmental processes, but also in plant responses to various environmental stress conditions. The involvement of NPC components in plant stress responses is mainly attributed to different mechanisms including control of mRNA/protein nucleo-cytoplasmic trafficking and transcriptional gene regulation. This mini review summarizes current knowledge of the NPC-mediated plant stress responses and provides an overview of the underlying molecular mechanisms.

Keywords: nuclear pore complex, nucleoporin, abiotic stress, biotic stress, nucleo-cytoplasmic transport

\section{INTRODUCTION}

The nuclear pore complex (NPC) is the gateway of macromolecular trafficking between the nucleus and the cytoplasm (Xu and Meier, 2008). Being one of the largest multi-protein complexes in the cell, the NPC consists of multiple copies of $\sim 30$ different proteins known as nucleoporins (Nups), which are organized in an octagonal manner and symmetrically around the cylindrical axis of the NPC (Alber et al., 2007; Tamura et al., 2010; Tamura and Hara-Nishimura, 2013). In addition to components that form the nuclear pore, importins and exportins that carry cargo proteins through the NPC gateway also belong to the NPC family according to the Transporter Classification Database (Saier et al., 2014). A brief overview of the plant NPC components and associated factors is shown in Figure 1A. In plants, the NPC family and their associated proteins have been shown to be involved in various biological processes such as responses to auxin, regulation of flowering time, abiotic stress responses, and defense responses to biotic stress (Bond, 2006; Dong et al., 2006b; Parry, 2014). This mini review focuses on the roles of the NPC in plant stress responses, providing an overview of known functions of the NPC and its associated factors in plant responses to abiotic and biotic stress conditions, followed by discussions on the underlying molecular mechanisms.

\section{INVOLVEMENTS OF NPC COMPONENTS IN PLANT STRESS RESPONSES}

The involvements of the NPC family components in plant stress responses were uncovered mostly by isolations of NPC mutants from forward genetic screenings. Diverse mechanisms have been 


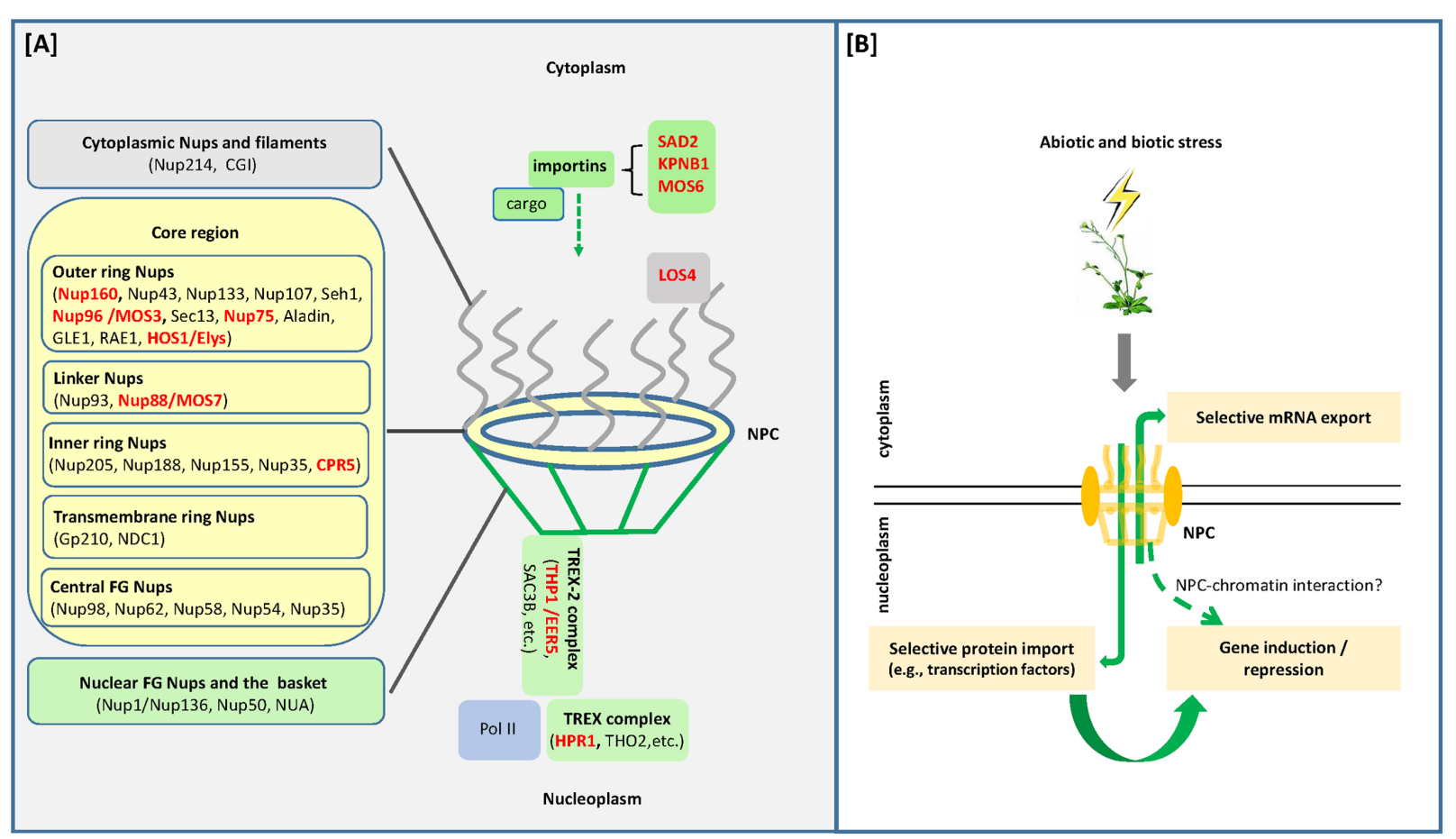

FIGURE 1 | (A) Schematic representation of the plant nuclear pore complex (NPC) and its associated factors. The NPC consists of the outer cytoplasm region, the symmetrical core region and the inner nucleoplasm region. The symmetrical core region is composed of the outer ring nucleoporins (Nups), the linker Nups, the inner ring Nups, the transmembrane ring Nups, and the central FG Nups (nucleoporins rich in phenylalanine-glycine repeats) (modified from Tamura and Hara-Nishimura, 2013). The nucleoplasm region is associated with the TREX-2 (transcription-coupled export 2) complex that, together with the TREX complex, couples Pol II transcription with mRNA export. Also shown are some importins and the LOS4 protein, which mediate protein/RNA nucleoplasmic trafficking as well as plant stress responses. Red names indicate proteins involved in plant stress responses. (B) The NPC functions in plant stress responses through diverse mechanisms. In plants under stress conditions, the NPC may selectively export stress-responsive mRNAs into the cytoplasm for protein synthesis; the NPC may also selectively import certain proteins such as stress-responsive transcription factors for transcriptional regulation; the NPC may also directly regulate gene expression at the transcriptional level through NPC-chromatin interactions.

proposed to interpret the dependence on various NPC family components for plant responses to different stress conditions, including cold, abscisic acid (ABA), drought, and biotic stress (Dong et al., 2006b; Verslues et al., 2006; Cheng et al., 2009; Luo et al., 2013). These findings clearly demonstrated the complexity of NPC-mediated plant stress responses. A brief summary of the NPC family components and NPC-associated factors known to be involved in plant stress responses is shown in Table $\mathbf{1}$.

\section{Cold Stress}

In plants, cold stress rapidly induces expression of many transcription factors, including the C-repeat-binding factors (CBFs), which activate transcription of various downstream cold-responsive (COR) genes (Chinnusamy et al., 2007; Zhu, 2016). Meanwhile, transcription of $C B F$ genes is controlled by their own upstream transcription factors, including the bHLH transcription factor ICE1 (INDUCER OF CBF EXPRESSION 1) (Zhu, 2016). In a genetic screen to search for mutations that impair cold-induced expression of the CBF3-LUC reporter gene, AtNUP160 was identified (Dong et al., 2006b). AtNUP160 protein is enriched in the nuclear rim and is critical for nucleo-cytoplasmic transport of mRNAs, as determined by
Poly (A)-mRNA in situ hybridization (Dong et al., 2006b). The atnup160-1 mutant plants displayed substantially reduced expression levels of $C B F$ genes, which have been shown to be important for acquired freezing tolerance (Gilmour et al., 2000; Lee et al., 2005). Consistent with the reduced induction of $C B F$ s, expression of cold-responsive genes were altered in the atnup160-1 mutant, accompanied by plant phenotypes of being sensitive to chilling stress and being defective in acquired freezing tolerance (Dong et al., 2006b).

In addition to AtNUP160, Arabidopsis HOS1 (high expression of osmotically responsive genes 1) is another clue for NPC involvement in plant stress responses. HOS1 was reported to be physically associated with the NPC components RAE1 (RNA export factor 1) and NUP43, as shown by immunoprecipitation in a proteomic study of plant NPCs (Tamura et al., 2010). Dysfunction of HOS1 resulted in over-accumulation of polyadenylated RNAs in the nucleus (MacGregor et al., 2013). In addition, HOS1 contains a region with homology to the vertebrate nucleoporin Elys (EMBRYONIC LARGE MOLECULE DERIVED FROM YOLK SAC) (Tamura et al., 2010), which is required for recruiting the Nup107-160 complex to chromatin (Gillespie et al., 2007; Doucet et al., 2010). Therefore HOS1 is considered as an NPC component in plants. 
TABLE 1 | Nucleoporins and the associated transport factors involved in Arabidopsis stress responses.

\begin{tabular}{|c|c|c|c|c|}
\hline Protein & Homologs & Stress & Phenotype & Reference \\
\hline NUP160 (SAR1) & Human NUP160 & Cold & $\begin{array}{l}\text { nup160 mutant is sensitive to chilling stress, } \\
\text { and defective in acquired freezing tolerance }\end{array}$ & Dong et al., 2006b \\
\hline HOS1 & Vertebrate Elys & Cold & $\begin{array}{l}\text { Overexpression of HOS1 confers increased } \\
\text { sensitivity to freezing stress }\end{array}$ & $\begin{array}{l}\text { Ishitani et al., 1998; } \\
\text { Lee et al., 2001; } \\
\text { Dong et al., 2006a }\end{array}$ \\
\hline LOS4 & Yeast DBP5 & Cold & $\begin{array}{l}\text { los } 4-1 \text { is more sensitive to chilling stress; los } 4-2 \\
\text { is more tolerant to chilling and freezing stress }\end{array}$ & $\begin{array}{l}\text { Gong et al., 2002, } \\
2005\end{array}$ \\
\hline SAD2 & Vertebrate Importin $\beta$ & ABA, UV-B & $\begin{array}{l}\text { sad2 mutant shows ABA hypersensitivity and } \\
\text { more tolerance to UV-B }\end{array}$ & $\begin{array}{l}\text { Verslues et al., } \\
\text { 2006; Zhao et al., } \\
2007\end{array}$ \\
\hline KPNB1 & Human Importin $\beta 1$ & ABA, drought & $\begin{array}{l}\text { atkpnb1 mutant showed ABA hypersensitivity } \\
\text { and enhanced drought tolerance }\end{array}$ & Luo et al., 2013 \\
\hline MOS3 & Human NUP96 & Biotic stress & $\begin{array}{l}\text { mos3 mutant exhibited enhanced disease } \\
\text { susceptibility to pathogens }\end{array}$ & Zhang and Li, 2005 \\
\hline MOS6 & Yeast Importin $\alpha 3$ & Biotic stress & $\begin{array}{l}\text { mos6 mutant exhibited enhanced disease } \\
\text { susceptibility to pathogens }\end{array}$ & Palma et al., 2005 \\
\hline MOS7 & Human NUP88 & Biotic stress & $\begin{array}{l}\text { mos } 7 \text { mutant plants exhibit defects in basal and } \\
\text { R protein-mediated immunity }\end{array}$ & Cheng et al., 2009 \\
\hline CPR5 & $\begin{array}{l}\text { None (a novel } \\
\text { transmembrane } \\
\text { nucleoporin) }\end{array}$ & Biotic stress & $\begin{array}{l}\text { Loss of function in CPR5 results in resistance } \\
\text { against pathogens; while overexpression } \\
\text { comprised the resistance }\end{array}$ & Gu et al., 2016 \\
\hline THP1 (EER5) & Yeast THP1 & Ethylene signaling & $\begin{array}{l}\text { The mutant showed enhanced ethylene } \\
\text { response }\end{array}$ & $\begin{array}{l}\text { Christians et al., } \\
2008\end{array}$ \\
\hline HPR1 & Yeast HPR1 & $\begin{array}{l}\text { Biotic stress and } \\
\text { ethylene signaling }\end{array}$ & $\begin{array}{l}\text { Mutation of hpr } 1 \text { suppress the EDR } 1 \text { mediated } \\
\text { disease resistance and enhance ethylene } \\
\text { induced senescence }\end{array}$ & $\begin{array}{l}\text { Pan et al., 2012; Xu } \\
\text { et al., } 2015\end{array}$ \\
\hline
\end{tabular}

Interestingly, Arabidopsis HOS1 was initially identified as a negative regulator in cold stress response from a genetic screening using the RD29A-LUC reporter system (Ishitani et al., 1997, 1998). In response to low temperature, the hos $1-1$ mutation causes enhanced induction of the CBF transcription factors and their downstream cold-responsive genes (Ishitani et al., 1998), while overexpression of HOS1 represses the expression of CBFs and their downstream genes as well as confers increased plant sensitivity to freezing stress (Dong et al., 2006a). HOS1 encodes an E3 ubiquitin ligase which accumulates in the nucleus in response to low temperatures (Lee et al., 2001). Cold stress also induces protein degradation of ICE1 that physically interacts with HOS1 (Dong et al., 2006a). Further investigation showed that HOS1 was required for the ubiquitination and degradation of ICE1 (Dong et al., 2006a). Since ICE1 is a transcription factor that positively regulates cold stress responses in Arabidopsis (Chinnusamy et al., 2003), it was proposed that HOS1 regulates plant cold stress responses through an ubiquitination proteasome pathway (Dong et al., 2006a).

Genetic screening using the RD29A:LUC reporter system has also identified LOS4 (low expression of osmotically responsive genes 4), which is essential for mRNA export (Gong et al., 2002). LOS4 encodes a DEAD-box RNA helicase, which is most closely related to the NPC-associated Dbp5p/Rat8p in yeast (Gong et al., 2002, 2005). The los4-1 mutant showed reduced gene expression levels of $C B F$ s and their downstream targets, as well as increased sensitivity to chilling stress (Gong et al., 2002). Interestingly, the same genetic screening later also isolated another los 4 allele, los 4 -2, which showed enhanced cold induction of CBF2 and its downstream target genes (Gong et al., 2005). In contrast to los $4-1$, the los $4-2$ mutant allele is more tolerant to chilling and freezing stresses, but is sensitive to heat stress (Gong et al., 2005). In situ poly(A) hybridization showed that the export of poly(A) RNAs was blocked in the los4-2 mutant at warm or high temperatures but not at low temperatures, whereas the los4-1 mutation weakened mRNA export at both low and warm temperatures (Gong et al., 2005), indicating that proper mRNA export is important for plant cold stress responses.

\section{ABA and Drought Stress}

Importin $\beta$ belongs to a large family of Importin $\beta$-like nuclear transport receptors that are also known as karyopherins (Gorlich and Kutay, 1999; Merkle, 2003). Proteins containing the importin $\beta$ domain can function as either importins that mediate nuclear protein import, or exportins that transport proteins out of the nucleus (Merkle, 2003). The Arabidopsis SAD2 (Super sensitive to $A B A$ and drought2) encodes an importin $\beta$-domain family protein and was identified in a genetic screening, which was based on alterations in the expression levels of the stress-responsive RD29A-LUC reporter gene (Verslues et al., 2006). The sad2-1 mutant showed increased luminescence after ABA, salt, cold or polyethylene glycol treatments, and exhibited ABA hypersensitivity in seed germination and seedling growth (Verslues et al., 2006). Although the mechanism underlying ABA hypersensitivity in sad2 is not clear, SAD2 may function either in importing a negative regulator of $\mathrm{ABA}$ response into the nucleus, or in exporting a positive regulator out of the 
nucleus, given that importin $\beta$ proteins can function as nuclear transport receptors. Indeed, the ability of SAD2 in transporting proteins was revealed later on. SAD2 was found to be required for nuclear import of MYB4, an R2R3-type transcription repressor that co-immunoprecipitated with SAD2 (Zhao et al., 2007). As a result of the absence of MYB4 protein in the nucleus, sad2 plants accumulated UV-absorbing pigments and displayed increased tolerance to UV-B radiation (Zhao et al., 2007).

To identify importin genes that function in drought tolerance, Luo et al. (2013) screened T-DNA insertion mutants of Arabidopsis importin $\beta$ family genes based on the ability to survive after drought treatment. A mutant with a T-DNA insertion in AtKPNB1 was identified to display considerably increased drought tolerance (Luo et al., 2013). AtKPNB1 encodes a homolog of human importin $\beta 1$, inactivation of which resulted in increased stomatal closure in response to ABA, lower rate of water loss, and substantially enhanced drought tolerance (Luo et al., 2013); while over-expression of AtKPNB1 led to increased sensitivity to drought compared to wild type plants, demonstrating that AtKPNB1 is an important negative effector of drought tolerance (Luo et al., 2013).

\section{Biotic Stress and Ethylene Response}

In addition to abiotic stress conditions, biotic stress can also trigger plant responses that involve the NPC. The Arabidopsis snc1 (suppressor of npr1-1, constitutive 1) mutant displays constitutive activation of disease resistance response against pathogens, due to a gain-of-function mutation in a TIR-NBS-LRR-type R gene (Zhang et al., 2003). Genetic screening for suppressors of $s n c 1$ isolated a series of double mutants named modifier of snc1 (mos), which no longer display constitutive resistance to virulent pathogens as the sncl single mutant (Palma et al., 2005; Zhang and Li, 2005). Among the MOS genes, MOS3, MOS6, and MOS7 encode proteins associated with the NPC. MOS3 encodes a protein with high sequence similarity with human nucleoporin96 (Zhang and Li, 2005). MOS6 encodes Arabidopsis importin a3 (Palma et al., 2005), while MOS7 is homologous to human and Drosophila melanogaster nucleoporin Nup88 (Cheng et al., 2009). Interestingly, it was found that nuclear accumulation of the defense signaling components EDS1 (Enhanced Disease Susceptibility 1) and NPR1 (Nonexpresser of PR genes 1) is significantly reduced in mos7-1 plants (Cheng et al., 2009). Moreover, CPR5 (Constitutive Expresser of Pathogenesis-Related Genes 5), which plays a key inhibitory role in effector-triggered immunity (ETI), was recently found to be a novel transmembrane nucleoporin (Wang et al., 2014; Gu et al., 2016). These findings clearly displayed an important role of nucleo-cytoplasmic trafficking in plant innate immunity.

Nuclear pore complex-dependent plant responses to biotic stress may involve ethylene signaling. In Nicotiana benthamiana, Nup75 (Nucleoporin 75) was identified as essential for plant resistance to Phytophthora infestans, and for the induction of some defense responses, including ethylene-mediated production of phytoalexin (Ohtsu et al., 2014). Arabidopsis HPR1 (HYPER RECOMBINATION1), which is a component of the NPC-associated TREX (Transcription-Export) complex, was isolated through a screening for edr1 (enhanced disease resistance) suppressors. In Arabidopsis, hprl mutation not only suppresses the enhanced disease resistance caused by edr1 mutation, but also increases ethylene-induced senescence in the edr1 background, suggesting that HPR1 plays a role in ethylene signaling pathway (Pan et al., 2012). It was also found that $h p r 1$ mutation suppresses plant insensitivity to ethylene as well as RTE1 (REVERSION-TO-ETHYLENE SENSITIVITY1) transcript levels in transgenic Arabidopsis that over-expressed RTE1 (Xu et al., 2015). An enhanced ethylene response was observed in of Arabidopsis eer5-1 mutant, which harbors a mutation in the NPC-associated TREX-2 component THP1 (Christians et al., 2008). Enhanced ethylene response in eer5-1 was correlated with failure to induce appropriately a subset of ethylene-regulated genes (Christians et al., 2008). Therefore, the NPC and its associated RNA export complexes may be involved in ethylene-mediated plant stress responses.

\section{MOLECULAR MECHANISMS OF NPC-DEPENDENT PLANT STRESS RESPONSES}

In eukaryotes, mRNAs synthesized in the nucleus need to be exported to the nucleoplasm for protein production; whereas nuclear proteins such as transcription factors must be imported, after protein synthesis in the cytoplasm, into the nucleus for proper function. Thus, roles of the NPC in plant stress responses are often attributed to NPC's function in controlling RNA/protein trafficking between the nucleus and the cytoplasm. In addition, it has also been shown that the NPC and its associated factors can be involved in certain biological processes through gene regulation at the transcriptional level.

\section{Selective mRNA Export}

By using in situ hybridization, many studies of nucleoporin mutants have demonstrated accumulation of polyadenylated mRNA in the nucleus (Dong et al., 2006b; Lu et al., 2010; Parry, 2014). Interestingly, stress conditions can also induce bulk mRNA accumulation in the nucleus (Saavedra et al., 1996; Bond, 2006; Muthuswamy and Meier, 2011). It is thus important to understand how certain mRNAs are selectively transported under stress conditions. In yeast, following heat or ethanol stress, poly(A) RNAs accumulates within nuclei, while mRNAs encoding Hsps (heat shock proteins) are efficiently exported from the nucleus (Saavedra et al., 1996; Bond, 2006). Recently, it was revealed that in yeast, cellular stress induces dissociation between regular mRNAs and the export receptor Mex67 as well as its adaptor proteins, thereby preventing general mRNA export; meanwhile, heat-shock mRNAs are efficiently exported in association with Mex67, without the need of adapter proteins (Zander et al., 2016). In fact, adaptor-bound mRNAs, but not free mRNAs, undergo quality control, indicating that at the cost of accuracy, heatshock mRNAs are exported and translated without delay, allowing cells to survive extreme situations (Zander et al., 2016). 
In Arabidopsis under heat or ethanol stress, a correlation seems to exist between altered protein sumoylation levels and bulk mRNA nuclear retention (Muthuswamy and Meier, 2011). Exposing Arabidopsis plants to heat shock and ethanol stress both resulted in elevation in high-molecular-weight SUMO conjugates, accompanied by nuclear mRNA accumulation (Muthuswamy and Meier, 2011). Because mutations in either SUMO E3 ligase SIZ1 or SUMO isopeptidase ESD4 (Early in short days 4) leads to nuclear mRNA retention, it has been suggested that sumoylation acts upstream of mRNA export, likely through the transient sumoylation status of one or more factors involved in mRNA trafficking (Muthuswamy and Meier, 2011).

\section{Control of Protein Transport}

Trafficking from the cytoplasm to the nucleus is essential for proteins with nucleus-specific functions. Under stress conditions, nuclear import of certain proteins can be critical for the plant to reprogram cellular processes to combat the stress. In Arabidopsis, MYB4 negatively regulate the transcription of cinnamate 4-hydroxylase $(\mathrm{C} 4 \mathrm{H})$ and thereby synthesis of sinapate esters which are UV-absorbing pigments (Zhao et al., 2007). MYB4 was found to co-immunoprecipitate with SAD2, which is an importin $\beta$-domain family protein essential for nuclear import of MYB4 (Zhao et al., 2007). Consistently, Arabidopsis sad2 mutant is more tolerant to UV-B radiation compared with wild type plants. Protein interaction between MYB4 and SAD2 requires the conserved GY/FDFLGL motif in the $\mathrm{C}$ terminus of MYB4, as demonstrated by the observation that an Asp to Asn mutation in the GY/FDFLGL motif abolishes the interaction between MYB4 and SAD2 (Zhou et al., 2015). Without a functional GY/FDFLGL motif, MYB4 failed to be transported into the nucleus and thus cannot repress their target genes (Zhou et al., 2015).

Besides SAD2, Arabidopsis MOS7 is another plant nucleoporin that has been shown to regulate nuclear accumulation of stress-responsive proteins. MOS7 is homologous to human and Drosophila nucleoporin Nup88 (Cheng et al., 2009). In animals, Nup88 attenuates NES (nuclear export signal)mediated protein nuclear export (Roth et al., 2003; Xylourgidis et al., 2006). In Arabidopsis, the mos7-1 mutation caused defects in basal and $\mathrm{R}$ protein-mediated immunity and in systemic acquired resistance (Cheng et al., 2009). Further investigation showed that nuclear accumulation of the autoactivated $\mathrm{R}$ protein snc1 as well as the defense signaling components EDS1 (Enhanced Disease Susceptibility 1) and NPR1 (Non-expresser of PR genes 1) was significantly reduced in mos $7-1$ plants, whereas nuclear abundance of other tested proteins was unaffected (Cheng et al., 2009), supporting the notion that trafficking of certain stress-responsive proteins can be subject to selective regulation by nucleoporins.

Despite the obvious specificity of the connection between the NPC and plant stress responses, little is known about how such specificity is achieved. Arabidopsis CPR5 (Constitutive Expresser of Pathogenesis-Related Genes 5) was initially identified as a negative regulator of plant Programmed Cell Death (PCD) and Effector-Triggered Immunity (ETI) (Boch et al., 1998; Wang et al., 2014). Loss-of-function mutations in CPR5 resulted in resistance against multiple pathogens, whereas over-expression of CPR5 compromised ETI-associated PCD and pathogen resistance in Arabidopsis (Boch et al., 1998; Wang et al., 2014). CPR5 was later revealed as a transmembrane nucleoporin (Wang et al., 2014; Gu et al., 2016). In addition, transient interference of CPR5 expression yielded in transcriptome patterns that significantly match plant responses to a variety of stress conditions including cold, salt/osmotic stress, abscisic acid, and various pathogens ( $\mathrm{Gu}$ et al., 2016). It was hypothesized that with compromised CPR5 function, the NPC adopts a structure with significantly increased permeability and/or transport activity that allows deregulated nuclear influx of diverse signaling cargos, which normally undergo nuclear translocation only under stimulus-induced conditions (Gu et al., 2016). Indeed, over-expression of CPR5 caused substantial cytoplasmic retention of NPR1, JAZ1, and ABI5, which are stress- and phytohormone-related nuclear proteins (Wang et al., 2014; Gu et al., 2016). Researchers further revealed that upon activation by immunoreceptors, CPR5 undergoes an oligomer-to-monomer conformational switch, which reconfigures the selective barrier to allow significant influx of nuclear signaling cargos through the NPC (Gu et al., 2016). These findings thus established CPR5 as a converging point in the specific connection between the NPC and the ETI/PCD responses in plants.

\section{Regulation of Gene Expression}

In addition to regulating RNA/protein transport, the NPC can also regulate gene expression at the transcriptional level. In as early as 1985, the "gene gating" hypothesis has proposed that certain expanded chromatin regions with transcription units can be attached to the NPC (Blobel, 1985). Subsequently this hypothesis has been supported by many studies in yeast and Drosophila. In yeast, several highly inducible genes are randomly distributed in the nucleoplasm when transcriptionally repressed but are recruited to the nuclear periphery upon activation (Brickner and Walter, 2004; Casolari et al., 2004; Taddei et al., 2006). In Drosophila, the nucleoporins Sec13, Nup98, and a subset of FG-repeat nucleoporins bind to developmentally regulated genes that are undergoing transcription induction (Capelson et al., 2010). Interestingly, certain NPC target genes exhibit transcriptional memory, i.e., after being repressed, these genes remain at the nuclear periphery for several generations and are primed for reactivation (Light et al., 2010). Transcriptional memory of yeast INO1 requires the NPC component Nup100, as well as incorporation of the histone variant $\mathrm{H} 2 \mathrm{~A} . \mathrm{Z}$ after gene repression (Light et al., 2010), indicating that the NPC can directly interact with chromatin and contribute to epigenetic gene regulation in response to developmental and environmental stimuli.

In plants, limited information is available for the mechanism of how the NPC regulates gene transcription. Arabidopsis hos 1 mutant exhibits an early flowering phenotype with repressed FLC gene expression. In an effort to explore how HOS1 regulates FLC (FLOWERING LOCUS C) expression, researchers (Jung et al., 2013) found that HOS1 binds to FLC chromatin in an FVE-dependent manner. In addition, HOS1 binding to the FLC 
locus is dramatically elevated at $4^{\circ} \mathrm{C}$. HOS 1 also interacts with the histone deacylase HDA6 and inhibits the binding of HDA6 to the FLC locus. Therefore, it was proposed that under short-term cold stress, HOS1 binds to FLC chromatin in an FVE-dependent manner to limit the chromatin accessibility to HDA6, allowing for activation of FLC transcription (Jung et al., 2013). Recently, our work isolated Arabidopsis SAC3B (SUPPRESSOR OF ACTIN3B), a core component of the TREX-2 (transcription-coupled export 2) complex, through a forward genetic screening for anti-silencing factors (Yang et al., 2017). Mutation of SAC3B caused gene silencing of a reporter gene luciferase driven by double 35S (d35S) promoter, accompanied by elevation in the repressive histone mark $\mathrm{H} 3 \mathrm{~K} 9 \mathrm{me} 2$ and by reduction in RNA polymerase Pol II occupancy (Yang et al., 2017). Moreover, THP1 (Tho2/Hpr1 PHENOTYPE1) and NUA (NUCLEAR PORE ANCHOR) were identified as SAC3B-associated proteins whose mutations also caused d35S::LUC silencing (Yang et al., 2017). The THP1 is another representative component of TREX-2 complex, while NUA is homologous to a component of nuclear pore basket in vertebrate (Krull et al., 2004; Jacob et al., 2007). Importantly, FLC gene expression is decreased in both $s a c 3 b$ and nua mutants (Xu et al., 2007; Yang et al., 2017), while our unpublished IP-MS results also identified HOS1 as a SAC3B-interacting protein. Thus it appears that the Arabidopsis NPC, in association with the TREX-2 complex, controls gene expression through HOS1-dependent chromatin targeting. In the future, it would be interesting to examine whether stress-inducible genes are tethered to the NPC, and whether stress memory can be mediated through chromatin tethering to the NPC.

\section{REFERENCES}

Alber, F., Dokudovskaya, S., Veenhoff, L. M., Zhang, W., Kipper, J., Devos, D., et al. (2007). The molecular architecture of the nuclear pore complex. Nature 450, 695-701. doi: 10.1038/nature06405

Blobel, G. (1985). Gene gating: a hypothesis. Proc. Natl. Acad. Sci. U.S.A. 82, 8527-8529. doi: 10.1073/pnas.82.24.8527

Boch, J., Verbsky, M. L., Robertson, T. L., Larkin, J. C., and Kunkel, B. N. (1998). Analysis of resistance gene-mediated defense responses in Arabidopsis thaliana plants carrying a mutation in CPR5. Mol. Plant Microbe Interact. 11, 1196-1206. doi: 10.1094/MPMI.1998.11.12.1196

Bond, U. (2006). Stressed out! Effects of environmental stress on mRNA metabolism. FEMS Yeast Res. 6, 160-170. doi: 10.1111/j.1567-1364.2006. 00032.x

Brickner, J. H., and Walter, P. (2004). Gene recruitment of the activated INO1 locus to the nuclear membrane. PLoS Biol. 2:e342. doi: 10.1371/journal.pbio.002 0342

Capelson, M., Liang, Y., Schulte, R., Mair, W., Wagner, U., and Hetzer, M. W. (2010). Chromatin-bound nuclear pore components regulate gene expression in higher eukaryotes. Cell 140, 372-383. doi: 10.1016/j.cell.2009.12.054

Casolari, J. M., Brown, C. R., Komili, S., West, J., Hieronymus, H., and Silver, P. A. (2004). Genome-wide localization of the nuclear transport machinery couples transcriptional status and nuclear organization. Cell 117, 427-439. doi: 10.1016/S0092-8674(04)00448-9

Cheng, Y. T., Germain, H., Wiermer, M., Bi, D. L., Xu, F., Garcia, A. V., et al. (2009). Nuclear pore complex component MOS7/Nup88 is required for innate immunity and nuclear accumulation of defense regulators in Arabidopsis. Plant Cell 21, 2503-2516. doi: 10.1105/tpc.108.064519

\section{CONCLUDING REMARKS}

It has been clear that a functional NPC is important to plants under stress conditions, and that involvements of the NPC family and the associated factors in plant stress responses can be mediated through different mechanisms including control of mRNA/protein trafficking and transcriptional gene regulation (Figure 1B). However, insights into the underlying mechanisms are still largely unclear. A key focus would be the target specificity of the NPC under stress conditions. It would also be important to understand how NPC components perceive stress signals, as well as to fully depict the involvement of NPC-dependent transcriptional regulation in plant stress responses.

\section{AUTHOR CONTRIBUTIONS}

YY and HZ drafted the manuscript. YY, WW, ZC, J-KZ, and HZ revised and finalized the manuscript.

\section{FUNDING}

$\mathrm{HZ}$ is supported by the Chinese Academy of Sciences.

\section{ACKNOWLEDGMENTS}

We apologize to those colleagues whose work is not cited due to space constraints. HZ is supported by the Chinese Academy of Sciences.

Chinnusamy, V., Ohta, M., Kanrar, S., Lee, B. H., Hong, X., Agarwal, M., et al. (2003). ICE1: a regulator of cold-induced transcriptome and freezing tolerance in Arabidopsis. Genes Dev. 17, 1043-1054. doi: 10.1101/gad.1077503

Chinnusamy, V., Zhu, J., and Zhu, J. K. (2007). Cold stress regulation of gene expression in plants. Trends Plant Sci. 12, 444-451. doi: 10.1016/j.tplants.2007. 07.002

Christians, M. J., Robles, L. M., Zeller, S. M., and Larsen, P. B. (2008). The eer5 mutation, which affects a novel proteasome-related subunit, indicates a prominent role for the COP9 signalosome in resetting the ethylene-signaling pathway in Arabidopsis. Plant J. 55, 467-477. doi: 10.1111/j.1365-313X.2008. 03521.x

Dong, C. H., Agarwal, M., Zhang, Y., Xie, Q., and Zhu, J. K. (2006a). The negative regulator of plant cold responses, HOS1, is a RING E3 ligase that mediates the ubiquitination and degradation of ICE1. Proc. Natl. Acad. Sci. U.S.A. 103, 8281-8286.

Dong, C. H., Hu, X. Y., Tang, W. P., Zheng, X. W., Kim, Y. S., Lee, B. H., et al. (2006b). A putative Arabidopsis nucleoporin, AtNUP160, is critical for RNA export and required for plant tolerance to cold stress. Mol. Cell. Biol. 26, 9533-9543.

Doucet, C. M., Talamas, J. A., and Hetzer, M. W. (2010). Cell cycle-dependent differences in nuclear pore complex assembly in metazoa. Cell 141, 1030-1041. doi: 10.1016/j.cell.2010.04.036

Gillespie, P. J., Khoudoli, G. A., Stewart, G., Swedlow, J. R., and Blow, J. J. (2007). ELYS/MEL-28 chromatin association coordinates nuclear pore complex assembly and replication licensing. Curr. Biol. 17, 1657-1662. doi: 10.1016/j. cub.2007.08.041

Gilmour, S. J., Sebolt, A. M., Salazar, M. P., Everard, J. D., and Thomashow, M. F. (2000). Overexpression of the Arabidopsis CBF3 transcriptional activator 
mimics multiple biochemical changes associated with cold acclimation. Plant Physiol. 124, 1854-1865. doi: 10.1104/pp.124.4.1854

Gong, Z. Z., Dong, C. H., Lee, H., Zhu, J. H., Xiong, L. M., Gong, D. M., et al. (2005). A DEAD box RNA helicase is essential for mRNA export and important for development and stress responses in Arabidopsis. Plant Cell 17, 256-267. doi: 10.1105/tpc.104.027557

Gong, Z. Z., Lee, H., Xiong, L. M., Jagendorf, A., Stevenson, B., and Zhu, J. K. (2002). RNA helicase-like protein as an early regulator of transcription factors for plant chilling and freezing tolerance. Proc. Natl. Acad. Sci. U.S.A. 99, 11507-11512. doi: 10.1073/pnas. 172399299

Gorlich, D., and Kutay, U. (1999). Transport between the cell nucleus and the cytoplasm. Annu. Rev. Cell. Dev. Biol. 15, 607-660. doi: 10.1146/annurev. cellbio.15.1.607

Gu, Y. N., Zebell, S. G., Liang, Z. Z., Wang, S., Kang, B. H., and Dong, X. N. (2016). Nuclear pore permeabilization is a convergent signaling event in effectortriggered immunity. Cell 166, 1526-1538. doi: 10.1016/j.cell.2016.07.042

Ishitani, M., Xiong, L., Lee, H., Stevenson, B., and Zhu, J. K. (1998). HOS1, a genetic locus involved in cold-responsive gene expression in arabidopsis. Plant Cell 10, 1151-1161. doi: 10.1105/tpc.10.7.1151

Ishitani, M., Xiong, L., Stevenson, B., and Zhu, J. K. (1997). Genetic analysis of osmotic and cold stress signal transduction in Arabidopsis: interactions and convergence of abscisic acid-dependent and abscisic acid-independent pathways. Plant Cell 9, 1935-1949. doi: 10.1105/tpc.9.11.1935

Jacob, Y., Mongkolsiriwatana, C., Veley, K. M., Kim, S. Y., and Michaels, S. D. (2007). The nuclear pore protein AtTPR is required for RNA homeostasis, flowering time, and auxin signaling. Plant Physiol. 144, 1383-1390. doi: $10.1104 /$ pp.107.100735

Jung, J. H., Park, J. H., Lee, S., To, T. K., Kim, J. M., Seki, M., et al. (2013). The cold signaling attenuator HIGH EXPRESSION OF OSMOTICALLY RESPONSIVE GENE1 activates FLOWERING LOCUS C transcription via chromatin remodeling under short-term cold stress in Arabidopsis. Plant Cell 25, 4378-4390. doi: 10.1105/tpc.113.118364

Krull, S., Thyberg, J., Bjorkroth, B., Rackwitz, H. R., and Cordes, V. C. (2004). Nucleoporins as components of the nuclear pore complex core structure and Tpr as the architectural element of the nuclear basket. Mol. Biol. Cell 15, 4261-4277. doi: 10.1091/mbc.E04-03-0165

Lee, B. H., Henderson, D. A., and Zhu, J. K. (2005). The Arabidopsis coldresponsive transcriptome and its regulation by ICE1. Plant Cell 17, 3155-3175. doi: $10.1105 /$ tpc.105.035568

Lee, H., Xiong, L., Gong, Z., Ishitani, M., Stevenson, B., and Zhu, J. K. (2001). The Arabidopsis HOS1 gene negatively regulates cold signal transduction and encodes a RING finger protein that displays cold-regulated nucleo-cytoplasmic partitioning. Genes Dev. 15, 912-924. doi: 10.1101/gad.866801

Light, W. H., Brickner, D. G., Brand, V. R., and Brickner, J. H. (2010). Interaction of a DNA zip code with the nuclear pore complex promotes $\mathrm{H} 2 \mathrm{~A} . \mathrm{Z}$ incorporation and INO1 transcriptional memory. Mol. Cell 40, 112-125. doi: 10.1016/j.molcel. 2010.09.007

Lu, Q., Tang, X., Tian, G., Wang, F., Liu, K., Nguyen, V., et al. (2010). Arabidopsis homolog of the yeast TREX-2 mRNA export complex: components and anchoring nucleoporin. Plant J. 61, 259-270. doi: 10.1111/j.1365-313X.2009. 04048.x

Luo, Y. J., Wang, Z. J., Ji, H. T., Fang, H., Wang, S. F., Tian, L. N., et al. (2013). An Arabidopsis homolog of importin 1 is required for ABA response and drought tolerance. Plant J. 75, 377-389. doi: 10.1111/tpj.12207

MacGregor, D. R., Gould, P., Foreman, J., Griffiths, J., Bird, S., Page, R., et al. (2013). HIGH EXPRESSION OF OSMOTICALLY RESPONSIVE GENES1 is required for circadian periodicity through the promotion of nucleo-cytoplasmic mRNA export in Arabidopsis. Plant Cell 25, 4391-4404. doi: 10.1105/tpc.113. 114959

Merkle, T. (2003). Nucleo-cytoplasmic partitioning of proteins in plants: implications for the regulation of environmental and developmental signalling. Curr. Genet. 44, 231-260. doi: 10.1007/s00294-003-0444-x

Muthuswamy, S., and Meier, I. (2011). Genetic and environmental changes in SUMO homeostasis lead to nuclear mRNA retention in plants. Planta 233, 201-208. doi: 10.1007/s00425-010-1278-7

Ohtsu, M., Shibata, Y., Ojika, M., Tamura, K., Hara-Nishimura, I., Mori, H., et al. (2014). Nucleoporin 75 is involved in the ethylene-mediated production of phytoalexin for the resistance of Nicotiana benthamiana to Phytophthora infestans. Mol. Plant Microbe Interact. 27, 1318-1330. doi: 10.1094/MPMI-0614-0181-R

Palma, K., Zhang, Y. L., and Li, X. (2005). An importin alpha homolog, MOS6, plays an important role in plant innate immunity. Curr. Biol. 15, 1129-1135. doi: 10.1016/j.cub.2005.05.022

Pan, H., Liu, S., and Tang, D. (2012). The THO/TREX complex functions in disease resistance in Arabidopsis. Plant Signal. Behav. 7, 422-424. doi: 10.4161/psb. 18991

Parry, G. (2014). Components of the Arabidopsis nuclear pore complex play multiple diverse roles in control of plant growth. J. Exp. Bot. 65, 6057-6067. doi: $10.1093 /$ jxb/eru346

Roth, P., Xylourgidis, N., Sabri, N., Uv, A., Fornerod, M., and Samakovlis, C. (2003). The Drosophila nucleoporin DNup88 localizes DNup214 and CRM1 on the nuclear envelope and attenuates NES-mediated nuclear export. J. Cell Biol. 163, 701-706. doi: 10.1083/jcb.200304046

Saavedra, C., Tung, K. S., Amberg, D. C., Hopper, A. K., and Cole, C. N. (1996). Regulation of mRNA export in response to stress in Saccharomyces cerevisiae. Genes Dev. 10, 1608-1620. doi: 10.1101/gad.10.13.1608

Saier, M. H., Reddy, V. S., Tamang, D. G., and Vastermark, A. (2014). The transporter classification database. Nucl. Acids Res. 42, D251-D258. doi: 10.1093/nar/gkt1097

Taddei, A., Van Houwe, G., Hediger, F., Kalck, V., Cubizolles, F., Schober, H., et al. (2006). Nuclear pore association confers optimal expression levels for an inducible yeast gene. Nature 441, 774-778. doi: 10.1038/nature 04845

Tamura, K., Fukao, Y., Iwamoto, M., Haraguchi, T., and Hara-Nishimura, I. (2010). Identification and characterization of nuclear pore complex components in Arabidopsis thaliana. Plant Cell 22, 4084-4097. doi: 10.1105/tpc.110.07 9947

Tamura, K., and Hara-Nishimura, I. (2013). The molecular architecture of the plant nuclear pore complex. J. Exp. Bot. 64, 823-832. doi: 10.1093/jxb/ers258

Verslues, P. E., Guo, Y., Dong, C. H., Ma, W., and Zhu, J. K. (2006). Mutation of SAD2, an importin beta-domain protein in Arabidopsis, alters abscisic acid sensitivity. Plant J. 47, 776-787. doi: 10.1111/j.1365-313X.2006.02833.x

Wang, S., Gu, Y. N., Zebell, S. G., Anderson, L. K., Wang, W., Mohan, R., et al. (2014). A noncanonical role for the CKI-RB-E2F cell-cycle signaling pathway in plant effector-triggered immunity. Cell Host Microbe 16, 787-794. doi: 10.1016/j.chom.2014.10.005

Xu, C. Y., Zhou, X., and Wen, C. K. (2015). HYPER RECOMBINATION1 of the THO/TREX complex plays a role in controlling transcription of the REVERSION-TO-ETHYLENE SENSITIVITY1 gene in Arabidopsis. PLoS Genet. 11:e1004956. doi: 10.1371/journal.pgen.1004956

Xu, X. M., and Meier, I. (2008). The nuclear pore comes to the fore. Trends Plant Sci. 13, 20-27. doi: 10.1016/j.tplants.2007.12.001

Xu, X. M., Rose, A., Muthuswamy, S., Jeong, S. Y., Venkatakrishnan, S., Zhao, Q., et al. (2007). NUCLEAR PORE ANCHOR, the Arabidopsis homolog of Tpr/Mlp1/Mlp2/megator, is involved in mRNA export and SUMO homeostasis and affects diverse aspects of plant development. Plant Cell 19, 1537-1548. doi: $10.1105 /$ tpc.106.049239

Xylourgidis, N., Roth, P., Sabri, N., Tsarouhas, V., and Samakovlis, C. (2006). The nucleoporin Nup214 sequesters CRM1 at the nuclear rim and modulates NFkappaB activation in Drosophila. J. Cell Sci. 119, 4409-4419. doi: 10.1242/jcs. 03201

Yang, Y., La, H., Tang, K., Miki, D., Yang, L., Wang, B., et al. (2017). SAC3B, a central component of the mRNA export complex TREX-2, is required for prevention of epigenetic gene silencing in Arabidopsis. Nucleic Acids Res. 45, 181-197. doi: 10.1093/nar/gkw850

Zander, G., Hackmann, A., Bender, L., Becker, D., Lingner, T., Salinas, G., et al. (2016). mRNA quality control is bypassed for immediate export of stressresponsive transcripts. Nature doi: 10.1038/nature20572 [Epub ahead of print].

Zhang, Y., Goritschnig, S., Dong, X., and Li, X. (2003). A gain-of-function mutation in a plant disease resistance gene leads to constitutive activation of downstream signal transduction pathways in suppressor of npr1-1, constitutive 1. Plant Cell 15, 2636-2646. doi: 10.1105/tpc.015842

Zhang, Y. L., and Li, X. (2005). A putative nucleoporin 96 is required for both basal defense and constitutive resistance responses mediated by suppressor of npr1-1, constitutive 1. Plant Cell 17, 1306-1316. doi: 10.1105/tpc.104.02 9926 
Zhao, J., Zhang, W., Zhao, Y., Gong, X., Guo, L., Zhu, G., et al. (2007). SAD2, an importin -like protein, is required for UV-B response in Arabidopsis by mediating MYB4 nuclear trafficking. Plant Cell 19, 3805-3818. doi: 10.1105/ tpc. 106.048900

Zhou, M., Sun, Z., Wang, C., Zhang, X., Tang, Y., Zhu, X., et al. (2015). Changing a conserved amino acid in R2R3-MYB transcription repressors results in cytoplasmic accumulation and abolishes their repressive activity in Arabidopsis. Plant J. 84, 395-403. doi: 10.1111/tpj.13008

Zhu, J. K. (2016). Abiotic stress signaling and responses in plants. Cell 167, 313-324. doi: 10.1016/j.cell.2016.08.029
Conflict of Interest Statement: The authors declare that the research was conducted in the absence of any commercial or financial relationships that could be construed as a potential conflict of interest.

Copyright (c) 2017 Yang, Wang, Chu, Zhu and Zhang. This is an open-access article distributed under the terms of the Creative Commons Attribution License (CC BY). The use, distribution or reproduction in other forums is permitted, provided the original author(s) or licensor are credited and that the original publication in this journal is cited, in accordance with accepted academic practice. No use, distribution or reproduction is permitted which does not comply with these terms. 